\section{Clonally related duodenal-type follicular lymphoma and in situ follicular neoplasia}

The genetic hallmark of follicular lymphoma (FL) is the translocation $\mathrm{t}(14 ; 18)$ (q32; $\mathrm{q} 21)$, which is found in up to $90 \%$ of $\mathrm{FL}$ and results in constitutive expression of the anti-apoptotic protein $\mathrm{Bcl} 2{ }^{1}$ The $\mathrm{t}(14 ; 18)+$ translocation is not sufficient to cause lymphoma, since $\mathrm{t}(14 ; 18)+\mathrm{B}$ lymphocytes can also be found in the peripheral blood of around $50 \%$ of healthy adults. ${ }^{2,3}$ In situ follicular neoplasia (ISFN) is a lymphoma precursor lesion considered to be a tissue manifestation of these circulating $t(14 ; 18)+B$ cells, which shows the genetic and immunophenotypic characteristics of FL, but is exclusively localized in the germinal centers (GC) of a morphologically reactive-appearing lymph node and by definition is only detectable by immunostaining for $\mathrm{Bcl} 2 ., 45$ The true incidence of ISFN is unknown, but studies of unselected lymph nodes from adults without evidence of lymphoma revealed a prevalence of $2-3 \%{ }^{6}$ The risk of progression to manifest FL seems to be low.

A similar proliferation of $\mathrm{t}(14 ; 18)+\mathrm{B}$ cells with limited malignant potential is duodenal-type follicular lymphoma (DFL), now considered as a distinct variant of FL in the 2016 update of the World Health Organization classification..$^{5}$ It shows overlapping characteristics with ISFN, including a low risk for progression to manifest FL even in the absence of specific treatment, and a lack of tumor mass formation. ${ }^{5,-9}$ Here we present a case of clonally related DFL and ISFN simultaneously present in the duodenal mucosa and peripancreatic lymph nodes found by chance in a Whipple resection specimen, respectively, documenting the close biological relationship of these two precursor lesions.

The case occurred in an 84-year-old man without a his-

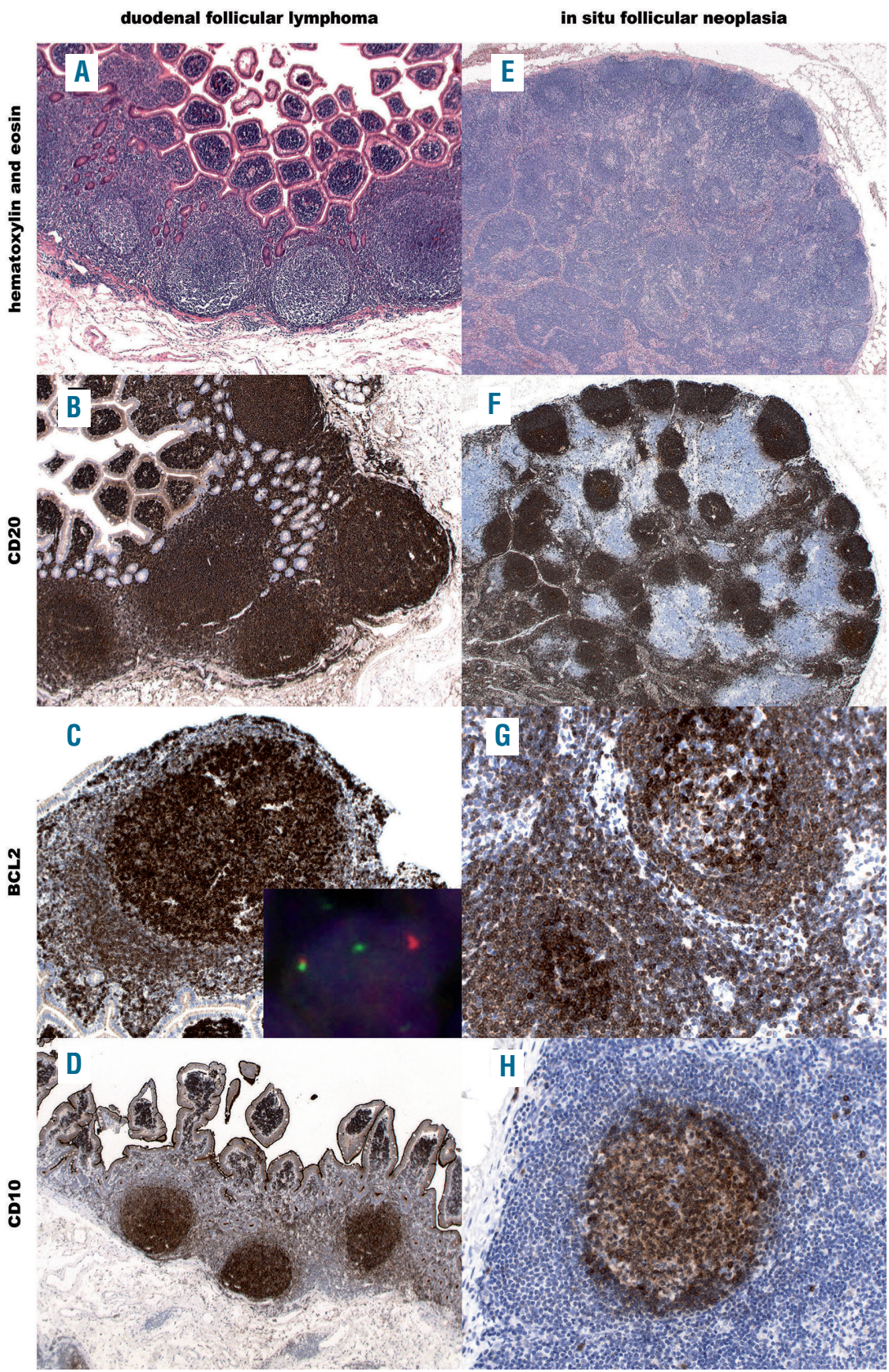

Figure 1. Morphological and immunophenotypical findings. A-D: Duodenal-type follicular lymphoma. A: Aggregates of neoplastic follicles restricted to the duodenal mucosa (hematoxylin and eosin, original magnification $\times 50)$. B: Strong and homogenous CD20 positivity in the follicular structures and in the surrounding area of the mucosa (CD20 immunoperoxidase, original magnification $\times 50)$. C: Tumor cells within the follicle with strong positivity for $\mathrm{Bcl} 2$ in comparison to the surrounding cells with weaker positivity (x100), inset: In fluorescence in situ hybridization (FISH) analysis, the cell shows a normal fusion signal and a separated red and green signal indicating a break. D: Strong positivity of the tumor cells within the follicles and the villi for CD10, the interfollicular area shows only scattered CD10 positive cells (x50). E-H: In situ follicular neoplasia (ISFN) in a lymph node. E: Lymph node with inconspicuous morphology with secondary follicles (hematoxylin and eosin, original magnification x25). F: Regular distribution of CD20 positive lymphocytes with many positive lymphocytes in the follicles and only few positive cells within perifollicular areas (x25). G: One germinal center with an almost complete replacement by strongly $\mathrm{Bcl} 2$ positive cells (left) and a follicle with only partial infiltration; note the weaker positivity of the reactive follicle mantle cells (x200). H: Typical strong CD10 positivity in a germinal center of ISFN, but virtually no CD10 positive cells outside the follicle (x200). Methods and technical procedures are described in detail in the Online Supplement. 


\section{ISFN}

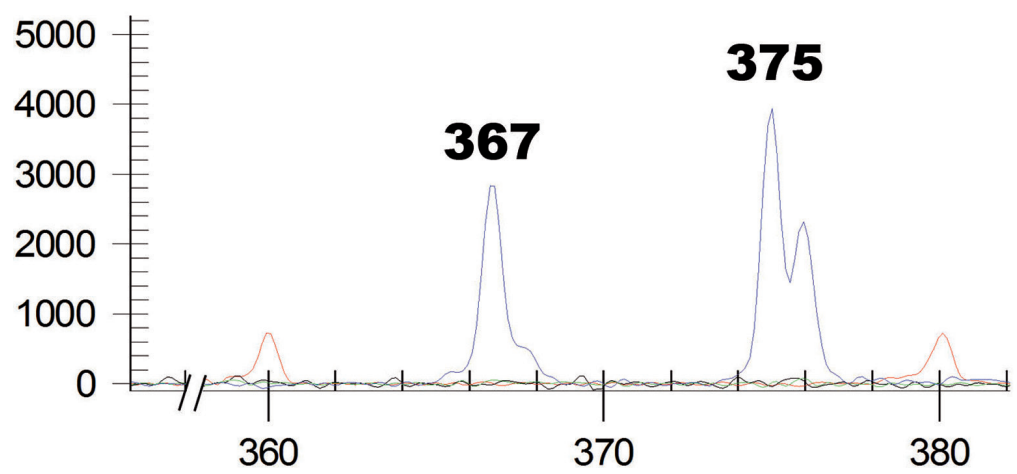

DFL

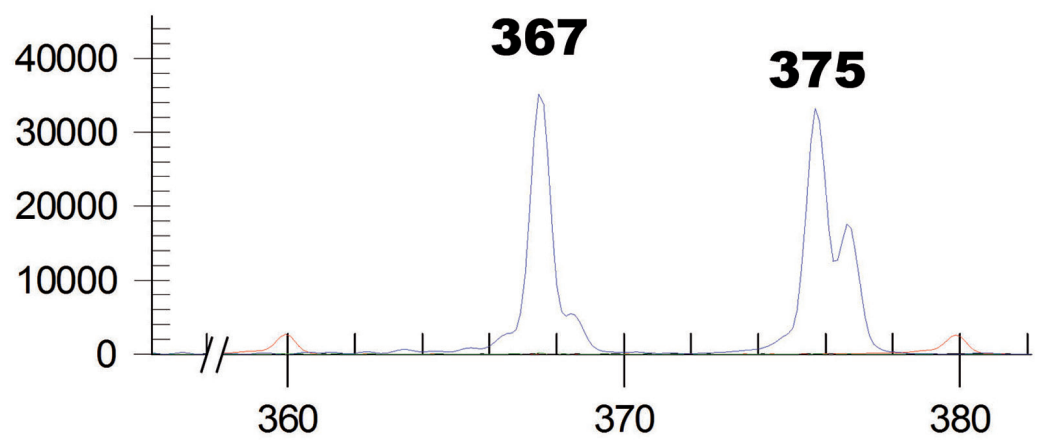

Figure 2. Molecular findings in a unique case of synchronous, clonally related in situ follicular neoplasia (ISFN) and duodenaltype follicular lymphoma (DFL). Detection of IGK rearrangements: Both the ISFN and the DFL show a clonal biallelic Vk/intron-Kde rearrangement with identical product lengths. Methods and technical procedures are described in detail in the Online Supplementary File.

tory of lymphoma who underwent resection of the pancreatic head, the duodenum and peripancreatic lymph nodes according to Whipple's procedure because of an adenoma of the ampullary region with a high grade intraepithelial neoplasia and the suspicion of ampullary carcinoma diagnosed on an endoscopic papillary biopsy. Imaging, including abdominal and pelvic computed tomography, as well as magnetic resonance imaging of the upper abdomen during the preoperative examination had shown a suspicious lesion in the ampullary region, but no evidence of pathological lymph nodes was found. Due to the patient's age, the inconspicuous preoperative lymph node status and an accompanying coronary artery disease and diabetes, a bone marrow examination was not performed. He has remained disease-free from lymphoma for the past four years since the resection.

Macroscopic inspection of the duodenal mucosa revealed multiple small nodules up to $1-2 \mathrm{~mm}$ in diameter, resembling follicular hyperplasia. The peripancreatic and periduodenal lymph nodes were not enlarged. Histopathological analysis of the duodenal mucosa revealed multiple lymphoid nodules consisting of single neoplastic follicles, sometimes back to back, but without the formation of larger lymphoid infiltrates (Figure 1A). There was a minimal spread into the upper parts of the submucosa, but no invasion of deeper parts. Cytologically, the follicles contained a monotonous proliferation of centrocytes with virtually no centroblasts and the absence of a distinct mantle zone. Immunohistochemically, the follicles, but also many peri-
Table 1. Non-synonymous mutations detected in duodenal-type follicular lymphoma.

\begin{tabular}{lccc}
\hline Mutation & Coding DNA & Protein level & Allelic frequency \\
KMT2D & c.8743C $>$ T & p.Arg2915Ter & $22 \%$ \\
EP300 & c.3479T $>$ C & p.Leu1160Pro & $12 \%$ \\
\hline STAT6 & c.1256A $>$ G & p.Asp419Gly & $20 \%$ \\
\hline
\end{tabular}

The mutations are specified on coding DNA, protein level, and allelic frequency. Methods and technical procedures are described in detail in the Online Supplementary File.

follicular lymphocytes within the villi expressed CD20 (Figure 1B) and Bcl6 and lacked CD5, CD23, and Cyclin D1. Intrafollicular cells revealed strong Bcl2 and CD10 positivity in comparison to the surrounding CD20 positive cells (Figure $1 \mathrm{C}-\mathrm{D})$. The proliferation rate assessed by MIB1 immunohistochemistry was generally low, but focally reached $20 \%$. IgD and CD23 staining revealed only focally a narrow and incomplete $\mathrm{IgD}$ and $\mathrm{CD} 23$ positive mantle zone. In addition, CD23 staining showed only follicular dendritic cell networks at the periphery of follicles, as typically observed in DFL.

All examined peripancreatic lymph nodes showed an inconspicuous morphology with secondary follicles and unremarkable CD20 staining (Figure $1 \mathrm{E}-\mathrm{F}$ ). Many germinal centers contained a variable amount of strongly Bcl2 and CD10 positive cells with co-expression of Bcl6 and a low proliferation rate, consistent with typical ISFN (Figure 
$1 \mathrm{G}-\mathrm{H})$. In contrast to the DFL, follicles were surrounded by a distinct IgD positive mantle zone. None of the examined lymph nodes revealed any manifestation of FL.

Fluorescence in situ hybridization (FISH) analysis using a break-apart probe for the BCL2 gene locus showed a break in BCL2 for the DFL (Figure 1C inset). Molecular studies were performed after macrodissection of both lesions. PCR for the immunoglobulin heavy chain (IGH) gene and IG kappa rearrangements demonstrated a polyclonal pattern with FR1-3 primers and an identical clonal, biallelic Vk/intron-Kde rearrangement in both the DFL and the ISFN (Figure 2), confirming a clonal relationship. Next-generation sequencing of the DFL using an Ion AmpliSeq Custom panel including 22 genes relevant for FL pathogenesis revealed missense mutations in KMT2D, STAT6 and EP300 (Table 1). The methods and technical procedures are described in detail in the Online Supplementary File.

Both ISFN and DFL are considered clinically indolent precursor/ early lesions of FL with a low risk of progression. They show similarities, but also clear differences, and the relationship between these two disorders is currently unknown. Whereas ISFN is a clinically precursor lesion identified by chance in lymph nodes resected for other purposes or in association with manifest lymphoma, ${ }^{6,10}$ DFL usually is detected endoscopically, given its characteristic appearance of multiple mucosal nodules as in this case. Another important distinction is the microarchitecture of DFL in comparison to ISFN. Whereas in classical ISFN, the accumulations of clonal $\mathrm{Bcl} 2^{+}$cells seem to be restricted to germinal centers with intact follicle mantles, suggesting colonization of pre-existent follicles by circulating $t(14 ; 18)+B$ cells, DFL likely represents a de novo formation of neoplastic follicles and usually reveals an identifiable extrafollicular tumor compartment. Recent genetic studies of ISFN and DFL have shown that in addition to the presence of the $t(14 ; 18)$ translocation, both already contain copy number aberrations and secondary mutations typical for FL, such as 1 p36 deletions and CREBBP, KMT2D, EZH2 and TNSFRSF14 mutations. ${ }^{711-13}$ Of note, mutations in KMT2D, EP300 and STAT6 identified in our case have all already been found in DFL, as well as in classical FL. ${ }^{7}$ Although their mutational landscape largely overlaps, FL and DFL differ significantly in their immune microenvironment and chemokine and cytokine milieu as assessed by gene expression profiling. ${ }^{7,14}$ DFL has phenotypical similarities to mucosa associated lymphoid tissue lymphoma, with an increased expression of CCL20 (chemokine ligand 20), and MAdCAM-1 (mucosal vascular addressin cell adhesion molecule 1), and shows a microenvironment characteristic of chronic inflammation. ${ }^{7,14}$ However, our case of clonally related DFL and ISFN in the same patient demonstrates that these differences may be more due to their distinct anatomical localization rather than intrinsic properties of tumor cells, since each lesion reproduced the microarchitectural features typical for their localization and thus confirms the hypothesis, that DFL most probably represents an FL in situ lesion of the gastrointestinal tract. ${ }^{7}$ Furthermore, this case provides evidence that these early clonal cells recirculate and may home to different compartments of lymphoid tissues. Although we were unable to trace cell migration and clonal architecture in our case due to the limited DNA quality and the lack of a detectable IGH rearrangement for the analysis of an ongoing somatic hypermutation, we have recently documented extensive interfollicular trafficking and ongoing IGH mutations in a case of ISFN with multiple involved nodes. $^{15}$
In conclusion, our case with clonally related, synchronously occurring ISFN and DFL re-enforces the hypothesis that both are different tissue manifestations of the same precursor-type lesion and that the presentation is probably more dependent on the microenvironment than on the genetic profile of the clonal population.

Dominik Nann, ${ }^{1}$ Irina Bonzheim, ${ }^{1}$ Inga Müller, ${ }^{1}$

Barbara Mankel,' Leticia Quintanilla-Martínez, ${ }^{1}$ Bence Sipos ${ }^{2}$ and Falko Fend'

Institute of Pathology and Neuropathology, University Hospital Tübingen and Comprehensive Cancer Center and ${ }^{2}$ Department of Internal Medicine VIII, University Hospital Tübingen, Tübingen, Germany

Correspondence: FALKO FEND

falko.fend@med.uni-tuebingen.de

doi:10.3324/haematol.2019.226142

Information on authorship, contributions, and financial \& other disclosures was provided by the authors and is available with the online version of this article at www. haematologica.org.

\section{References}

1. Horsman DE, Gascoyne RD, Coupland RW, Coldman AJ, Adomat SA. Comparison of cytogenetic analysis, southern analysis, and polymerase chain reaction for the detection of $\mathrm{t}(14 ; 18)$ in follicular lymphoma. Am J Clin Pathol. 1995;103(4):472-478.

2. Limpens J, Stad R, Vos C, et al. Lymphoma-associated translocation $\mathrm{t}(14 ; 18)$ in blood $\mathrm{B}$ cells of normal individuals. Blood. 1995;85(9):2528-2536.

3. Dolken G, Illerhaus G, Hirt C, Mertelsmann R. BCL-2/JH rearrangements in circulating $\mathrm{B}$ cells of healthy blood donors and patients with nonmalignant diseases. J Clin Oncol. 1996;14(4):1333-1344.

4. Cong P, Raffeld M, Teruya-Feldstein J, Sorbara L, Pittaluga S, Jaffe ES. In situ localization of follicular lymphoma: description and analysis by laser capture microdissection. Blood. 2002;99(9):33763382 .

5. Jaffe ES, Harris NL, Swerdlow SH, et al. Follicular lymphoma. In: Swerdlow SH, Campo E, Harris NL, Jaffe ES, Pileri SA, Stein H, et al., eds. WHO Classification of Tumors of the Haematopoietic and Lymphoid Tissues. Revised 4th ed ed. Lyon: IARC Press, 2017:266277.

6. Henopp T, Quintanilla-Martinez L, Fend F, Adam P. Prevalence of follicular lymphoma in situ in consecutively analysed reactive lymph nodes. Histopathology. 2011;59(1):139-142.

7. Hellmuth JC, Louissaint A, Jr., Szczepanowski M, et al. Duodenaltype and nodal follicular lymphomas differ by their immune microenvironment rather than their mutation profiles. Blood. 2018;132(16):1695-1702

8. Mamessier E, Broussais-Guillaumot F, Chetaille B, et al. Nature and importance of follicular lymphoma precursors. Haematologica. 2014;99(5):802-810.

9. Schmatz AI, Streubel B, Kretschmer-Chott E, et al. Primary follicular lymphoma of the duodenum is a distinct mucosal/submucosal variant of follicular lymphoma: a retrospective study of 63 cases. J Clin Oncol. 2011;29(11):1445-1451.

10. Montes-Moreno S, Castro Y, Rodriguez-Pinilla SM, et al. Intrafollicular neoplasia/in situ follicular lymphoma: review of a series of 13 cases. Histopathology. 2010;56(5):658-662.

11. Mamessier E, Song JY, Eberle FC, et al. Early lesions of follicular lymphoma: a genetic perspective. Haematologica. 2014;99(3):481488.

12. Schmidt J, Ramis-Zaldivar JE, Bonzheim I, et al. CREBBP gene mutations are frequently detected in in situ follicular neoplasia. Blood. 2018;132(25):2687-2690.

13. Schmidt J, Salaverria I, Haake A, et al. Increasing genomic and epigenomic complexity in the clonal evolution from in situ to manifest $\mathrm{t}(14 ; 18)$-positive follicular lymphoma. Leukemia. 2014;28(5):1103-1112.

14. Takata K, Tanino M, Ennishi D, et al. Duodenal follicular lymphoma: comprehensive gene expression analysis with insights into pathogenesis. Cancer Sci. 2014;105(5):608-615.

15. Kosmidis P, Bonzheim I, Dufke C, et al. Next generation sequencing of the clonal IGH rearrangement detects ongoing mutations and interfollicular trafficking in in situ follicular neoplasia. PloS One. 2017;12(6):e0178503. 